\title{
Polarization patterns of the twilight sky
}

Thomas W. Cronin, Eric J. Warrant, Birgit Greiner

Thomas W. Cronin, Eric J. Warrant, Birgit Greiner, "Polarization patterns of the twilight sky," Proc. SPIE 5888, Polarization Science and Remote Sensing II, 58880R (18 August 2005); doi: 10.1117/12.613053

SPIE. Event: Optics and Photonics 2005, 2005, San Diego, California, United States 


\title{
Polarization patterns of the twilight sky
}

\author{
Thomas W. Cronin ${ }^{* a}$, Eric J. Warrant ${ }^{\mathrm{b}}$, Birgit Greiner ${ }^{\mathrm{b}}$ \\ ${ }^{a}$ Dept. of Biological Sciences, University of Maryland Baltimore County, Baltimore, MD, \\ USA 21250 \\ ${ }^{\mathrm{b}}$ Dept. of Cell and Organism Biology, Lund University, Helgonavägen 3, 22362 Lund, Sweden
}

\begin{abstract}
Although natural light sources produce depolarized light, patterns of partially linearly polarized light appear in the sky due to scattering from air molecules, dust, and aerosols. Many animals, including bees and ants, orient themselves to patterns of polarization that are present in daytime skies, when the intensity is high and skylight polarization is strong and predictable. The halicitid bee Megalopta genalis inhabits rainforests in Central America. Unlike typical bees, it forages before sunrise and after sunset, when light intensities under the forest canopy are very low, and must find its way to food sources and return to its nest in visually challenging circumstances. An important cue for the orientation could be patterns of polarization in the twilight sky. Therefore, we used a calibrated digital camera to image skylight polarization in an overhead patch of sky, $87.6^{\circ}$ across, before dawn on Barro Colorado Island in Panama, where the bees are found. We simultaneously measured the spectral properties of polarized light in a cloudless patch of sky $15^{\circ}$ across centered on the zenith. We also performed full-sky imaging of polarization before dawn and after dusk on Lizard Island in Australia, another tropical island. During twilight, celestial polarized light occurs in a wide band stretching perpendicular to the location of the hidden sun and reaching typical degrees of polarization near $80 \%$ at wavelengths $>600 \mathrm{~nm}$. This pattern appears about 45 minutes before local sunrise or disappears 45 minutes after local sunset (about 20 minutes after the onset of astronomical twilight at dawn, or before its end at dusk) and extends with little change through the entire twilight period. Such a strong and reliable orientation cue could be used for flight orientation by any animal with polarization sensitivity that navigates during twilight.
\end{abstract}

Keywords: polarized light, polarization sensitivity, polarization vision, celestial polarization, twilight, nocturnal bee

\section{INTRODUCTION}

Most animals, mainly invertebrate species but also some vertebrates, have photoreceptors that respond differentially depending on the orientation of the e-vector of incoming polarized light. Many species have incorporated this polarization sensitivity into visual systems that at least partially analyze the properties of the polarization stimulus, extracting information about the degree and orientation of polarization. This information can be used to analyze the properties of surfaces, to recognize objects, and to recognize particular biological signals produced using patterns of polarized light. Polarization vision can also be involved in systems of orientation and navigation, whereby animals use naturally occurring patterns of polarization that appear in the sky to orient themselves when foraging and possibly even when migrating. Known cases of polarization orientation involve celestial polarization produced by the presence in the sky of the sun or moon, but there is good evidence that some animals can orient effectively at times when the sun is below the horizon. Celestial patterns of polarization during twilight have not been previously described.

\subsection{Polarized light in nature}

No important natural light source emits polarized light. Yet polarized light is abundant in nature, being produced by purely physical processes such as atmospheric scattering or by reflection from dielectric surfaces as well as by biological structures specialized to be efficient reflectors or even emitters of linear or circular polarization. The patterns produced in the sky are highly predictable (though changeable throughout the day) and thus usable for animal orientation ${ }^{1}$, particularly in air. The general appearance of the celestial polarization pattern can be visualized as a hemispherical field, centered on the sun, with e-vector orientations always perpendicular to the plane defined by the position of the sun (or moon), the point of scattering, and the location of the observer. While nearly all the scattered light is polarized to some degree, the maximum degree of polarization is observed along the great circle at $90^{\circ}$ from the light source (typically the sun, but this same pattern exists in moonlight $)^{2,3,4}$. Maximum polarization varies with sky state, being greatest when the *cronin@umbc.edu; phone 1410 455-3449; fax 1410 455-3875 
sky is clear and dry, but the form of the pattern itself depends only on the position of the light source (sun or moon). This pattern is observable even in open sky patches when the source is occluded by clouds.

\subsection{Animal orientation using polarized light patterns}

Because the pattern of polarized light in the sky is fully determined by the location of the sun, it can serve as a compass for animal orientation, provided only that the animal in question is capable of detecting and analyzing polarized light, and also able to interpret its pattern throughout the celestial field. The benefit of using the polarization rather than the sun (or moon) directly is that small patches of unobscured sky are sufficient for the task, permitting excellent orientation even when the sun is invisible. It turns out that many animals use celestial polarization patterns for orientation. By far, the best examples are found among insects ${ }^{1,3,5}$. Typically, day-active insects use specialized photoreceptors found in the dorsal regions of their compound eyes to inspect the polarization field around the zenith, orienting themselves using a time-compensated compass ${ }^{5,7}$. Strategies used by such insects have successfully been implemented in autonomous robotic systems ${ }^{8,9}$. Less familiar are the abilities of some insects (e.g. dung beetles) to orient using nocturnal polarization patterns created by the scattering of moonlight ${ }^{10,11}$. The moonlight patterns are essentially identical to those generated by sunlight ${ }^{4}$. Full-sky imaging, generally using fisheye optics, has now been carried out under the open daylight $\mathrm{sky}^{2,3}$, in moonlight ${ }^{4}$, and under clouds and the forest canopy ${ }^{12}$.

In the cases just cited, the light source producing the celestial polarization pattern actually appears in the sky. We have recently become interested in celestial patterns of polarization that occur when the sun or moon is not present in the sky. These patterns are produced during the time period between the onset (or end) of astronomical twilight and sunrise (or sunset). Astronomical twilight commences in the morning when the sun's influence is just detectable in the upper atmosphere, by convention when the sun's center is $18^{\circ}$ below the eastern horizon. Obviously, the reverse situation holds in the evening. The duration of astronomical twilight depends on the inclination of the sun's path in a given situation; in the tropics, where the sun moves nearly perpendicularly to the horizon most of the year, it lasts $\sim 1.25$ to $\sim 1.5 \mathrm{~h}$. During the earlier parts of morning astronomical twilight, the influence of the sun is barely perceptible, but since it directionally illuminates the upper atmosphere, polarized light is scattered to the earth's surface.

Despite the minimal illumination provided by skylight near the extremes of astronomical twilight, some flying insects can navigate at these times. The halictid bee Megalopta genalis emerges from its nests in the Panamanian forest beginning about 1 hour before sunrise and forages for 15 to 30 minutes, probably on flowers in the forest canopy, before returning accurately to its home ${ }^{13}$. The bee repeats this pattern in reverse at sunset. Its eyes have specializations for extreme dim-light vision ${ }^{13,14}$ and polarization sensitivity (Greiner et al., in prep), and it is likely that this animal makes use of the upper-atmospheric polarization pattern during its foraging flights. While a serious challenge to the visual system, the use of polarization cues by bees has advantages during this period. Since the sun is moving nearly perpendicularly to the horizon, its azimuth changes very slowly over the period before twilight, making for a stable polarization pattern. Extremely wide-field polarization sensors, like those of nocturnal crickets ${ }^{7}$ (animals that are capable of polarization orientation at nocturnal skylight intensities), would be effective for such a task and would have the advantage of good performance even when much of the sky is obscured by forest canopy or by clouds. Invertebrates are apparently not the only animals that utilize the twilight polarization pattern for orientation. Nocturnally migrating birds become disoriented when provided with a depolarized celestial pattern during twilight ${ }^{15}$.

We have begun to characterize the spatial and spectral features of twilight celestial polarization using imaging and spectral polarimetric techniques. By doing so, we hope to understand how night-flying animals successfully orient in such demanding conditions. Polarization imaging of the night sky (at times when a polarization pattern is present) could be useful for certain applications. Objects in the sky, such as clouds, stars, satellites, aircraft, high-flying balloons, etc. will not normally be polarized, or their polarization will be unlike that of the skylight pattern. Such objects could be visualized in real-time using a sensitive polarization imaging system optimized for the special conditions of twilight.

\section{MATERIALS AND METHODS}

Partially linearly polarized light is defined by three parameters at each wavelength: intensity, degree of polarization (\% polarization), and $e$-vector angle. Accordingly, three measurements are required for the full analysis of this light. We acquired separate measurements at $0^{\circ}, 45^{\circ}$, and $90^{\circ}$ in our work. Photographs of a patch of sky $\left(67^{\circ} \times 55^{\circ}\right)$ centering on 
the zenith were made at a location near the habitat of Megalopta genalis at the Smithsonian Tropical Research Institute, Barro Colorado Island, Panama, from 0520 through 0605 (sunrise at 0609) on April 17, 2004. The photographs were acquired using an Olympus C-5050Z digital camera with a wide-angle lens attachment, with a linear polarizing filter placed over the lens. Simultaneously with the photographic series, spectral measurements of polarization were acquired using an Ocean Optics USB2000 spectrometer sampling though a $15^{\circ}$ aperture looking directly at the zenith. Full-sky measurements of twilight polarization were made at Lizard Island Research Station, Queensland, Australia at dusk (1800-2000, sunset at 1814) on September 14 and 15, 2004 and at dawn (0415-0630, sunrise at 0610 and 0608) on September 19 and 23, 2004. For this work we used a Nikon Coolpix 5700 digital camera with a fisheye lens attachment imaging a field slightly larger than a hemisphere. The polarizing filter was placed inside the tube that attached the fisheye lens to the camera, such that it could be rotated to the desired angles. The technique was checked by imaging a large sheet of polaroid material, and no obvious optical distortions or decreases in polarization were noted throughout the field. All data were analyzed according to Wolff and Andreou ${ }^{16}$, after first correcting each digital photograph for the response of the detector. Both cameras were calibrated individually for their response-intensity functions using a grayscale target (Macbeth Color Checker) photographed over a range of exposures. Pixel values in each color band $(\mathrm{R}, \mathrm{G}, \mathrm{B})$ from 0 to 255 could then be weighted for linearity.

\section{RESULTS}

\subsection{Full-sky measurements}

The earliest appearance of photographed polarization is illustrated in Figure 1, which shows selected images from a series acquired at sunrise at the Australian site on September 19, 2004. In all panels of these full-sky images (Figs. 1, 2, and 3) the zenith is centered in the image, $\mathrm{N}$ is directly upwards, and $\mathrm{E}$ is to the left (opposite to the card of a compass, since the camera is looking upwards). Sunrise itself was at 0610 on this day, but a low hill to the east obscured the sun until after the measurement series was concluded. At this time and location, astronomical twilight (the time when the sun's influence just begins to be detectable in the sky, taken to occur when the sun's center is $18^{\circ}$ below the horizon) commenced at 0459. Photographs were taken from 0415 through 0630, but the first suggestion of polarization seen photographically appeared at 0515 (Fig. 1). Images in Fig. 1 (particularly at 0500 and 0515) were taken through a polarizing filter oriented $\mathrm{E} / \mathrm{W}$ and are strongly contrast-enhanced to show the suggestion of a band of polarization running $\mathrm{N} / \mathrm{S}$ that is just apparent at 0515 , about 15 minutes after the onset of astronomical twilight.

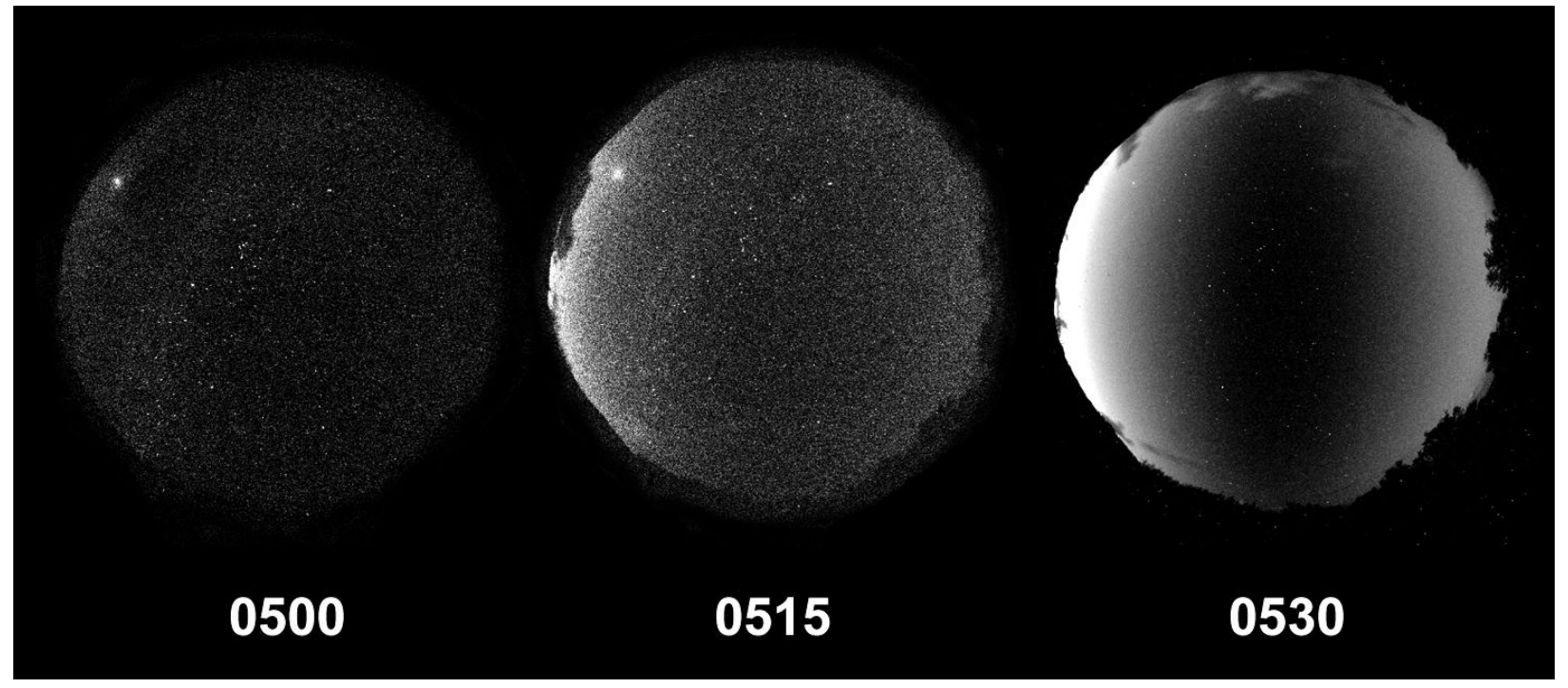

Figure 1: Polarization of the pre-dawn sky. These photographs, strongly contrast-enhanced, were taken before sunrise on September 19, 2004 at Lizard Island, Australia with a polarizing filter oriented E/W. Time of sunrise: 0610; astronomical twilight; 0459. In all panels, the zenith is in the center, $\mathrm{N}$ is to the top and $\mathrm{E}$ to the left. The bright "star" in the east is Venus; Orion is nearly overhead. Many constellations are visible (and more in the original photographs) as well as the Milky Way extending along the eastern sky. 


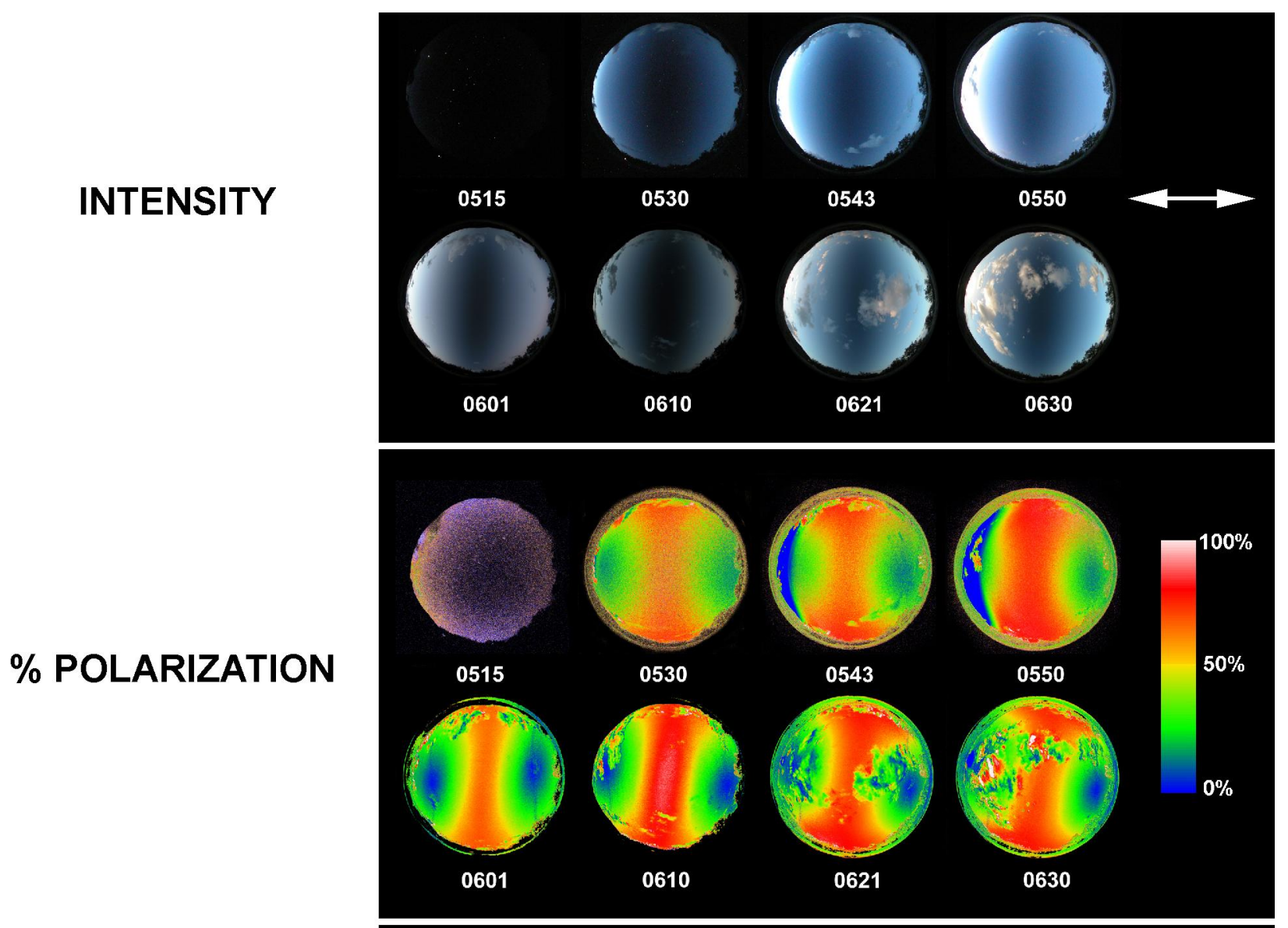

\section{\% POLARIZATION}

\section{e-VECTOR ANGLE}

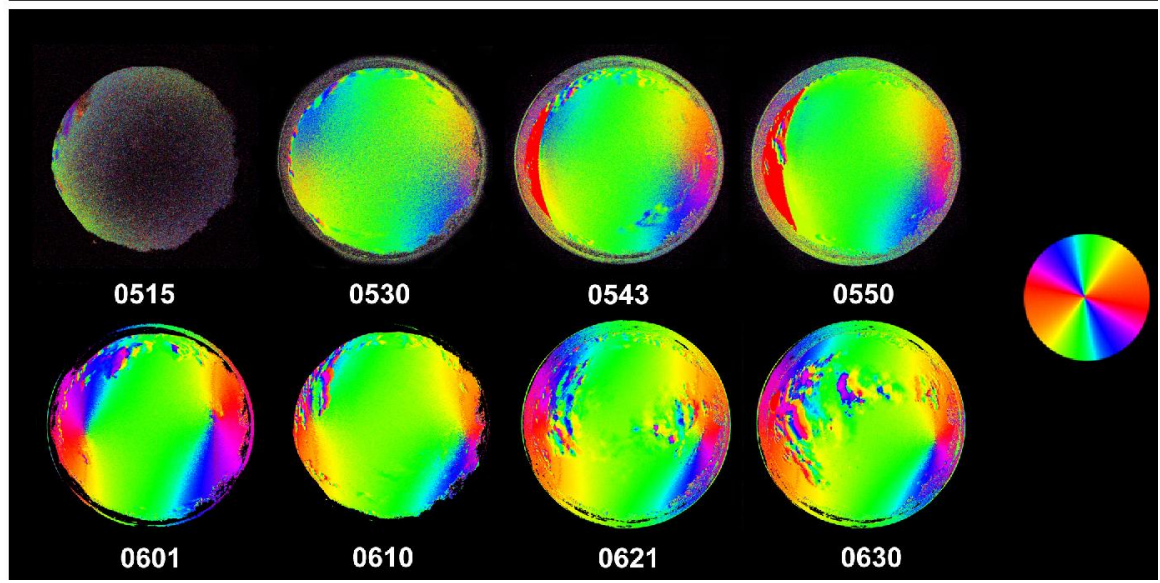

Figure 2: Polarization of the pre-dawn and dawn sky. Full-sky images were acquired using a Nikon Coolpix 5700 digital camera with a fisheye lens attachment having a linear polarizing filter mounted between the lens and the camera itself, and polarization series of images were acquired as described in the text. Data in this figure are from the same series as depicted in Fig. 1, on September 19, 2004 at Lizard Island, Australia (sunrise: 0610; astronomical twilight: 0459). Photographs were taken from 0415 through 0630, but no analyzable polarization was detected before 0515 , so earlier data are not displayed. The top set of images are originals acquired when the polarizing filter was oriented $\mathrm{E} / \mathrm{W}$ (indicated by the double-headed arrow, emphasizing the N/S $e$-vector orientation). The middle set shows analyzed \% polarization in false color, encoded as displayed in the key to the right. The bottom set indicates $e$-vector angle, also in false color as coded in the key to the right. The odd color patterns in the images of $\%$ polarization and $e$-vector angle are caused by clouds moving between images in original sets of exposures. 
Original photographs and fully analyzed sets of images are seen in Figure 2. Although we began acquiring images at 0415 , no polarization was detectable by analysis before 0530 , so Fig. 2 depicts data from 0515 through 0630 . The top set displays original TIFF images seen when the polarizer was directed $\mathrm{E} / \mathrm{W}$, thus emphasizing the actual pattern of celestial polarization (which primarily has N/S-directed $e$-vectors). The images at 0515 and 0530 are the same as seen in Fig. 1, but are in real color and are not enhanced here.) The middle panel set shows degree of polarization (\% polarization) coded in false color, as indicated in the key to the right. Starting at 0530 (as early as 0520 in other series, but the set illustrated in Fig. 2 did not include images taken at that time) a clear band of strong polarization, typically exceeding $50 \%$, runs across the vault of the sky nearly directly from $\mathrm{N}$ to $\mathrm{S}$. Once established, the pattern of polarization is remarkably stable. This is also emphasized by the stable pattern of the distribution of polarization angle ( $e$-vector angle), displayed in the bottom set of panels. Here, again, angle is coded in false color, with the key on the right; the color displayed is that of the diameter extending in the same direction. As expected, the band of greatest polarization is polarized with its axis oriented N/S, and since the light source is placed horizontally to the E, the pattern of angles (like the pattern of $\%$ polarization) has a mirror-like symmetry - note that the $e$-vector angles proceed around the horizon in the same sequence as the colors of the key, showing a regular shift in polarization angle with azimuth.

As expected, celestial polarization proceeds through a series of changes at dusk mirroring those seen at dawn. Figure 3 shows a series acquired on September 14, 2004 at the Lizard Island site. Sunset was at 1814, with astronomical twilight terminating at 1925 . On this occasion, the degree of polarization was generally less than in any of the other three series, either at dawn or dusk, and the last time when measurable polarization was seen was 1850, about 35 minutes after sunset. In the other sunset series, polarization continued to be measurable until 1900, 45 minutes after sunset. Angles of polarization were identical to those of the dawn series, and were constant throughout the evening twilight (data not shown). Of course, these data were obtained within a week of the autumnal equinox, near the equator (latitude $-14^{\circ} 40^{\prime}$ ), so sunrise and sunset were nearly perfectly symmetrical.

Figure 3: Polarization of the sky at sunset and during evening twilight. These images are from analyzed data acquired on September 14, 2004 at Lizard Island, and show the degree of polarization as coded in the key at the bottom. Time of sunset: 1814; astronomical twilight; 1925. In all panels, the zenith is in the center, $\mathrm{N}$ is to the top and $\mathrm{E}$ to the left. Note that the strong $\mathrm{N} / \mathrm{S}$ pattern of polarization remains near $50 \%$ polarized until $\sim 1850,36$ minutes after sunset.

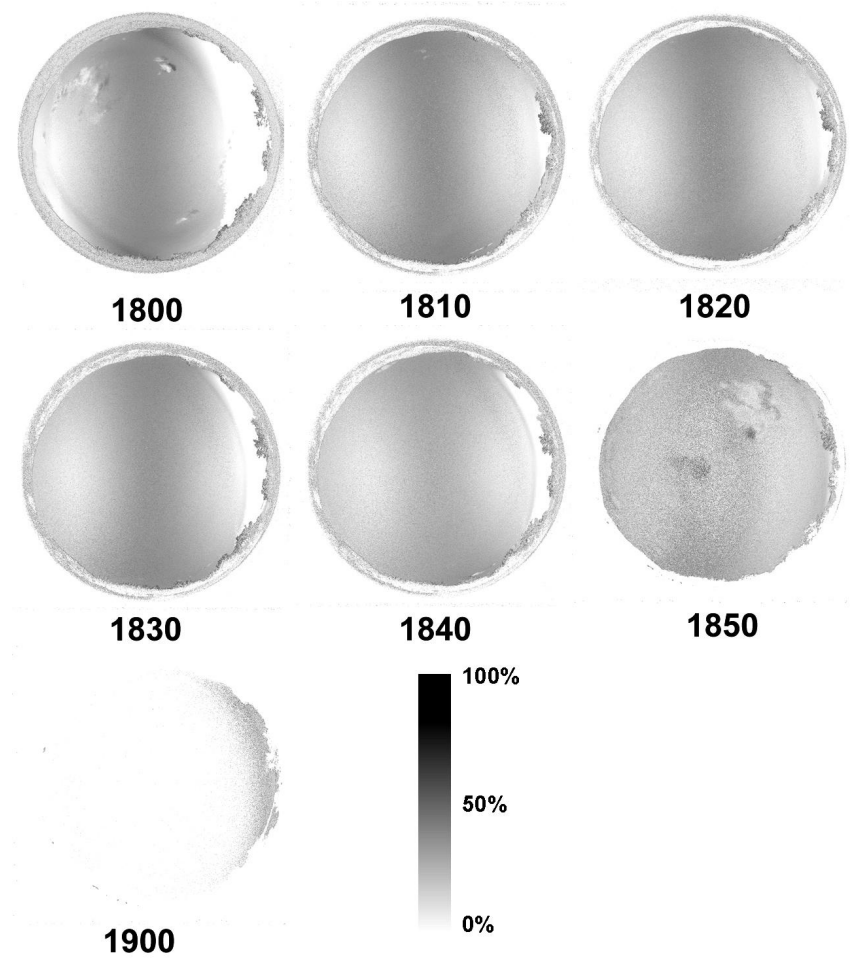

\subsection{Spectral characteristics of twilight polarization}

Rayleigh scattering that produces celestial polarization is greatest at short wavelengths, but skylight has its greatest polarization at middle to long wavelengths ${ }^{17,18}$. This is because the initially high polarization content in the ultraviolet is rapidly degraded by multiple-path scattering as the light makes its way to the earth's surface. In fact, one reason that 
insects may use ultraviolet photoreceptors to detect polarization is to enhance their ability to analyze polarized light that has been scattered nearer to their eyes, beneath clouds or under the forest canopy ${ }^{18}$. Since the polarization seen before dawn must originate high in the atmosphere, where sunlight is being scattered at first twilight, one might expect overall low values of polarization and an even greater deterioration of short-wavelength polarization than that seen during the day. This would be predicted because the scattered, polarized light must traverse the entire lower atmosphere on its way to an observer, providing ample opportunity for multiple scattering and loss of polarization content. During one dawn twilight sequence on April 17, 2004, at Barro Colorado Island in Panama, we were able to measure full spectral features of polarization in a $15^{\circ}$ patch of sky centered on the zenith throughout the period from 0540 to 0604 (sunrise: 0609; beginning of astronomical twilight; 0457). While it would have been desirable to have a data series extending earlier into the twilight period, the spectrometer configuration we used was not sensitive enough for this, so the data illustrate spectral polarization from about 20 minutes before until 5 minutes before sunrise. Figure 4 shows the degree of polarization throughout this period. Percent polarization increased gradually and continuously from $\sim 60 \%$ at $350 \mathrm{~nm}$ to $\sim 75 \%$ at $750 \mathrm{~nm}$; there is a suggestion of increasing degree of polarization with time as well, but the change is slight and very gradual. Certainly, at the time of the earliest measurement (0540), polarization is about $10 \%$ less than at later times (a similar pattern can be seen in the analogous panels of Fig. 2). It would be quite interesting to observe the polarization spectrum at yet earlier times. The angle of polarization was constant (at about $5^{\circ} \mathrm{W}$ of $\mathrm{N}$ ) throughout the entire measurement series and showed no variation with wavelength, as expected.

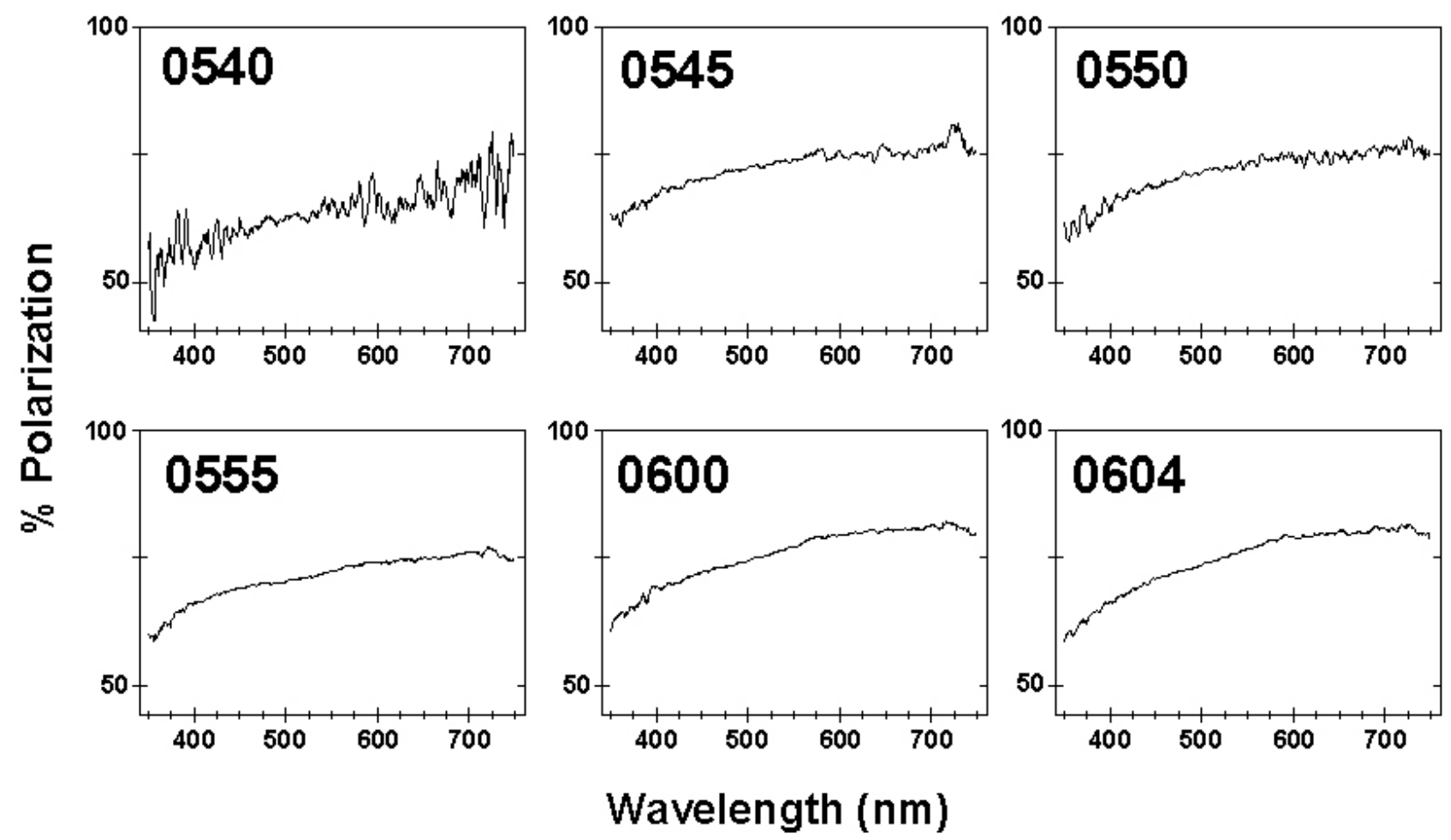

Figure 4: Polarization spectra of the pre-dawn sky. These data were collected before dawn on April 17, 2004 at Barro Colorado Island, Panama. Dawn: 0606; Astronomical twilight: 0457. The time of measurement is given on each panel. Note that the polarization spectrum was fairly constant from the first time when measurements were possible, showing a gradual increase in degree of polarization from about $60 \%$ at $350 \mathrm{~nm}$ to about $75 \%$ at $750 \mathrm{~nm}$.

\subsection{Presence of non-polarized objects in the twilight sky}

Artificial and natural objects in the sky during twilight can be very difficult to detect, locate, and identify. Such objects could include clouds, patches of haze, satellites, high-flying balloons, or aircraft. Polarization of these will almost always differ from that of the skylight around them (even if they are above the atmosphere), making them far more visible when using polarization imaging. Objects that are at low to moderate altitudes and are of similar brightness to the background skylight are particularly susceptible to being emphasized using polarization imaging, while objects at 
greater heights are more difficult to separate using this technique. Figure 5 illustrates a patch of pre-dawn sky centered on the zenith seen in intensity (I, left panel; greatly contrast-enhanced) and in degree of polarization (P, right panel); the white arrows indicates a star and the black one, a patch of hazy cloud. Both the star and cloud are visible in both panels, but the polarization image shows both more clearly, and other patches of haze are visible as blotches that reduce the degree of polarization of skylight are visible throughout the polarization image. The polarization image has another benefit. The brightness gradation across the intensity image (caused by the influence of the sun below the horizon) is removed when the scene is analyzed only for polarization, emphasizing differences due to changes in polarization content alone.
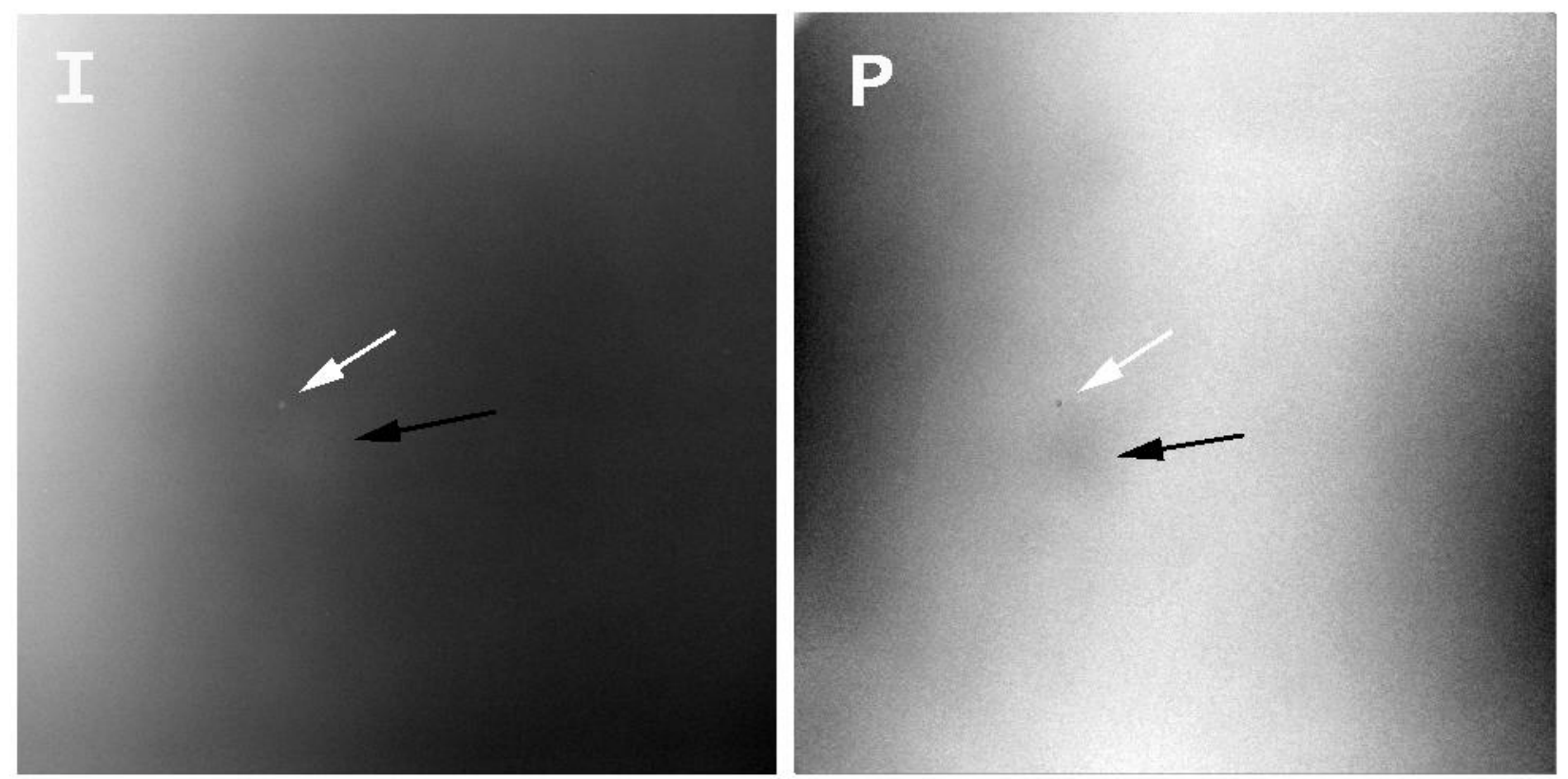

Figure 5: Images of objects in the pre-dawn sky. These images were collected before dawn, at 0540 on April 17, 2004 at Barro Colorado Island, Panama. Dawn: 0606; Astronomical twilight: 0457. The images are centered on the zenith; north is to the top, east to the left. The panel to the left (I) shows intensity, strongly contrast-enhanced for small variations. The right panel (P) shows degree of polarization, with greater brightness indicating higher $\%$ polarization. The white arrow indicates the star Altair, seen as a bright (luminous) point in the intensity image and a dark (depolarized) point in the polarization image. The black arrow shows a patch of cloud faintly visible in the intensity image and obvious as a dark blotch in the polarization image. A number of other patches of haze are visible in the polarization image as darker patches, but these are difficult or impossible to see in the intensity image.

\section{SUMMARY AND CONCLUSIONS}

The presence of polarization in the daytime sky has long been recognized, and for some time it has been known that various animals, both vertebrates and invertebrates, use the pattern of this polarization as a compass cue for orientation. While the daytime pattern of celestial polarization is fully predictable and strong, it shows complex changes throughout the day as the sun travels across the sky. Similar problems face animals that use nocturnal polarization patterns generated by moonlight. In contrast, during the period from near the onset of astronomical twilight to dawn (or the reverse at dusk), celestial polarization has a very constant pattern, varying little in angular distribution, degree of polarization, or spectral content throughout much of this time. Hence, for animals capable of detecting skylight polarization at very low intensities, the twilight interval provides a relatively simple orientation cue in the heavens. Usable polarized light appears in the sky for about a 1-hour interval between the margins of astronomical twilight and actual sunrise or sunset. Skylight polarization at this time may also be useful for technical applications, in that it provides a passive way to detect, localize, and potentially identify difficult-to-see objects in the sky. We are enthusiastic about further investigations of twilight polarization and plan to use instrumentation with greater sensitivity at lower light levels to track the twilight polarization pattern deeper into the night. 


\section{ACKNOWLEDGEMENTS}

We thank the staff of the Smithsonian Tropical Research Institute at Barro Colorado Island and the Lizard Island Research Station for support and assistance. This work is based on research supported by the National Science Foundation under Grant Number IBN-023582, by the Air Force Office of Scientific Research under Grant Number 02NL253, and by grants from the Swedish Research Council. Jamie Theobald assisted in the collection of polarization spectra.

\section{REFERENCES}

1. R. Wehner, "Polarization vision - a uniform sensory capacity?", Journal of Experimental Biology, 204, 2589-2596, 2001.

2. K.J. Voss and Y. Liu, "Polarized radiance distribution measurements of skylight. I. System description and characterization", Applied Optics, 36, 6083-6094, 1997.

3. G. Horváth and D. Varjú D, Polarized Light in Animal Vision. Berlin, Heidelberg, New York: Springer, 2004.

4. J. Gál, G. Horváth, A. Barta and R. Wehner, "Polarization of the moonlit clear night sky measured by full-sky imaging polarimetry at full moon: comparison of the polarization of moonlit and sunlit skies", Journal of Geophysical Research D, 106, 22647-22653, 2001.

5. R. Wehner, "The ant's celestial compass system: spectral and polarizational channels", Orientation and Communication in Arthropods, M. Lehrer (ed.), Basel: Birkhäuser Verlag, 145-185, 1997.

6. S. Rossel, "Polarization sensitivity in compound eyes", Facets of Vision, D.G. Stavenga and R.C. Hardie (eds), Springer, Berlin, pp. 298-316, 1989.

7. T. Labhart, J. Petzold, and H. Helbling, "Spatial integration in polarization-sensitive interneurones of crickets: a survey of evidence, mechanisms and benefits", Journal of Experimental Biology, 204, 2423-2430, 2001.

8. D. Lambrinos, H. Kobayashi, R. Pfeifer, M. Maris, T. Labhart, and R. Wehner, "An autonomous agent navigating with a polarized light compass", Adaptive Behavior, 6 , 131-161, 1997.

9. D. Lambrinos, R. Möller, T. Labhart, R. Pfeifer, and R. Wehner, "A mobile robot employing insect strategies for navigation", Robotics and Autonomous Systems, 30, 39-64, 2000.

10. M. Dacke, D.E. Nilsson, C.H. Scholtz, M. Byrne, and E.J. Warrant, "Insect orientation to polarized moonlight", Nature, 424, 33, 2003.

11. M. Dacke, D.E. Nilsson, C.H. Scholtz, M. Byrne, and E.J. Warrant, "Lunar orientation in a beetle", Proceedings of the Royal Society of London B, 271, 361-365, 2004.

12. I. Pomozi, G. Horváth, and R. Wehner, "How the clear-sky angle of polarization pattern continues underneath clouds: full-sky measurements and implications for animal orientation", Journal of Experimental Biology, 204, 2933-2942, 2001.

13. E.J. Warrant, A. Kelber, A. Gislen, B. Greiner, W. Ribi, and W. Wcislo, "Nocturnal vision and landmark orientation in a tropical halictid bee", Current Biology, 14, 1309-1318, 2004.

14. B. Greiner, W.A. Ribi, and E.J. Warrant, "Retinal and optical adaptations for nocturnal vision in the halictid bee Megalopta genalis". Cell and Tissue Research, 316, 377-390, 2004.

15. A.J. Helbig, "Depolarization of natural skylight disrupts orientation of an avian nocturnal migrant", Experientia, 46, 755-758, 1990.

16. L.B. Wolff and A.G. Andreou, "Polarization camera sensors", Image and Vision Computing, 13, 497-510, 1995.

17. K.L. Coulson, Polarization and Intensity of Light in the Atmosphere, A. Deepak Publishing, Hampton, VA, USA, 1988.

18. A. Barta and G. Horváth, "Why is it advantageous for animals to detect celestial polarization in the ultraviolet? Skylight polarization under clouds and canopies is strongest in the UV", Journal of Theoretical Biology, 226, 429437, 2004. 